\title{
Nanoscale Communication with Molecular Arrays in Nanonetworks
}

\author{
Baris Atakan, Member, IEEE, Sebastià Galmés, Member, IEEE, and Ozgur B. Akan, Senior Member, IEEE
}

\begin{abstract}
Molecular communication is a promising nanoscale communication paradigm that enables nanomachines to exchange information by using molecules as communication carrier. Up to now, the molecular communication channel between a transmitter nanomachine (TN) and a receiver nanomachine (RN) has been modeled as either concentration channel or timing channel. However, these channel models necessitate exact time synchronization of the nanomachines and provide a relatively low communication bandwidth. In this paper, the Molecular ARray-based COmmunication (MARCO) scheme is proposed, in which the transmission order of different molecules is used to convey molecular information without any need for time synchronization. The MARCO channel model is first theoretically derived, and the inter-symbol interference and error probabilities are obtained. Based on the error probability, achievable communication rates are analytically obtained. Numerical results and performance comparisons reveal that MARCO provides significantly higher communication rate, i.e., on the scale of $100 \mathrm{Kbps}$, than the previously proposed molecular communication models without any need for synchronization. More specifically, MARCO can provide more than $250 \mathrm{Kbps}$ of molecular communication rate if inter-symbol time and inter-node distance are set to $2 \mu \mathrm{s}$ and $2 \mathrm{~nm}$, respectively.
\end{abstract}

Index Terms-Molecular communications, nanonetworks, molecular order, molecular array, synchronization-free molecular communication.

\section{INTRODUCTION}

Nanotechnology envisages the practical realization of very low-end nanomachines that have tiny components to accomplish a simple specific task such as communication, computation, and sensing in a scale ranging from 1 to 100 nanometers. For example, molecular machines, a particular type of nanomachines, consist of organized molecular components to achieve a specific task as a response to an external stimulus [1]. Nanomachines are considered as a part of the potential solution approaches to many crucial problems, e.g., pollution, scarce food, and cellular repair [2].

While a single nanomachine can perform a simple specific task, a network of communicating nanomachines can also be engineered to share nanoscale information over a nanonetwork so as to fulfill complex tasks such as collaborative drug delivery, health monitoring, and biological or chemical attack detection [3], [4]. In fact, such nature-made nanonetworks already exist and are indispensable to cooperatively share nanoscale information for a specific task. For example, the quorum

B. Atakan and O. B. Akan are with Next-generation Wireless Communications Laboratory at Department of Electrical and Electronics Engineering in Koç University, İstanbul, Turkey, 34450, Email: \{batakan, akan\}@ku.edu.tr.

S. Galmés is with Department of Mathematics and Computer Science in University of Balearic Islands, Palma de Mallorca, Spain, 07122, Email: sebastia.galmes@uib.es. sensing mechanism allows bacteria to communicate with each other by producing, emitting, and receiving hormone-like messenger molecules called autoinducer. This natural process permits bacteria to synchronize all colony activities and to change the colony state as a response to an external stimulus [5]. Similar to bacteria, in natural immune system, the white blood cells called B-cells and T-cells communicate with each other using molecules to cooperatively sense and eliminate the hazardous pathogen [6]. The molecular communication among white blood cells forms the biological immune network that is an excellent defense mechanism of the organisms.

As in nature, nanomachines can also be artificially interconnected via molecular communication to constitute a nanonetwork for a specific task [4]. For example, two artificial cell-to-cell communication systems for yeast are developed in [7]. In the first system, the engineered yeasts, considered as sender cells, synthesize and emit the plant hormone cytokinin and the emitted cytokinin molecules diffuse into the environment and activate a target signaling pathway in nearby engineered yeasts, considered as receiver cells. In the second system, like quorum sensing in bacteria, the molecular communication between sender and receiver cells provides population density-dependent gene expression process for the synthesis of a functional biochemical material. These artificial cell-to-cell communication systems are expected to provide new biotechnology applications such as biomaterial fabrication and tissue engineering.

Besides the realization of artificial molecular communication systems, there also exist several research efforts toward addressing the unique challenges of molecular communications by the viewpoint of communication theory. In [8], the concept of molecular communication is introduced and the first attempt for design of molecular communication system is performed. An extensive survey on nanonetworking with molecular communication is presented in [3]. A physical channel model is introduced by incorporating three different phases of molecular communication, i.e., molecular emission, diffusion, and reception, into a single channel model [9]. Achievable information rates are investigated for the molecular communication channel, which is modeled as a timing channel in [10]. Transmission time of the emitted molecules are considered as the input to the timing channel and time delay experienced in delivery of molecules is also a random noise of the timing channel.

The molecular noise in diffusion-based molecular communication systems is investigated and modeled in [11]. The effect of noise on the molecular communication performance is examined by considering various molecular transmission, 
propagation, encoding, and decoding mechanisms [12]. An information theoretical analysis is proposed to reveal the exact dynamics of the relay channel and find out its communication capacity [13]. In-sequence delivery of different molecules in a molecular communication channel is explored and different transmission strategies are proposed [14]. However, this work does not analyze the capabilities of in-sequence molecular delivery by providing a comprehensive analysis with the derivation of the inter-symbol interference and error probability of the channel.

In [15], we introduced an information theoretical approach for molecular communication, derived a closed-form expression for the capacity of molecular communication channel and proposed an adaptive error compensation technique for molecular communication. We also provided the deterministic capacity of information flow in a molecular nanonetwork [16], and explored the necessity of networking techniques for the realization of future nanonetworks. We also modeled the pointto-point and multiple-access molecular channels and discuss their achievable molecular communication rates in [17] and [18]. However, we have not yet considered molecular arraybased communication in any of our previous work.

All of these existing and previously developed molecular channel models between a transmitter nanomachine (TN) and a receiver nanomachine ( $\mathrm{RN}$ ) have been considered as either concentration channel or timing channel. However, these channel models require exact time synchronization between $\mathrm{TN}$ and RN, which is beyond the state-of-the-art capabilities of very low-end nanomachines [19]. Furthermore, they provide a relatively low communication bandwidth, which may hamper some frontier applications such as molecular processor with molecular inter/intra-chip communication. Therefore, it is imperative for the realization of future nanonetworks to develop synchronization-free and practical molecular communication schemes providing relatively high communication bandwidth. Hence, in order to address this need, in this paper, we introduce the Molecular ARray-based COmmunication (MARCO) scheme in which the transmission order of different molecules is used to convey molecular information without any need for time synchronization between $\mathrm{TN}$ and $\mathrm{RN}$. Unlike the previous channel models, MARCO also follows an efficient approach in which information is encoded by the arrays of different molecules. This also resembles the natural encoding of genetic information, i.e., DNA arrays consisting of different base pairs. We first theoretically model MARCO channel behavior. Then, we comprehensively derive channel characteristics such as inter-symbol interference and error probabilities based on the principles of Brownian motion. Using these probabilities, we finally obtain a communication rate expression for the MARCO channel. Numerical results and performance comparisons reveal that MARCO provides significantly higher communication rate, i.e., on the scale of $100 \mathrm{Kbps}$, than the previously proposed molecular communication schemes without any need for time synchronization. More specifically, MARCO can provide more than $250 \mathrm{Kbps}$ of molecular communication rate if inter-symbol time and inter-node distance are set to $2 \mu \mathrm{s}$ and $2 \mathrm{~nm}$, respectively.

The remainder of this paper is organized as follows. In

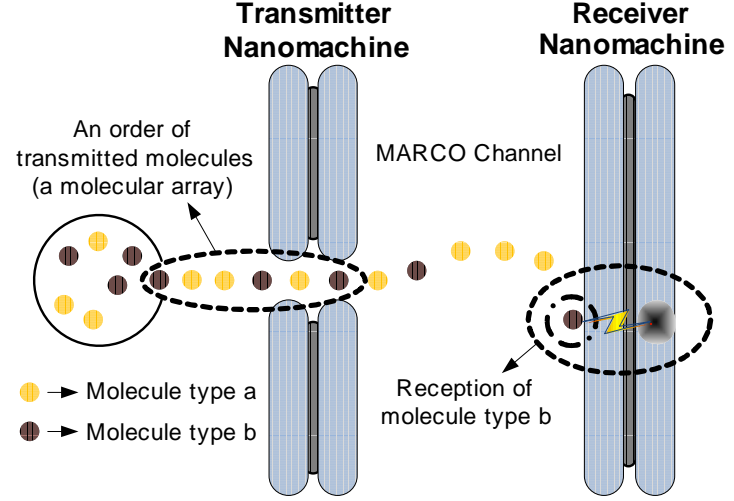

Fig. 1. Illustration of molecular order-based communication. Here, two distinct molecules, i.e., molecule type $a$ and $b$, are used to transmit information.

Section II, we present the MARCO scheme and derive the inter-symbol interference and error probabilities based on which a rate expression for the MARCO channel is given. In Section III, we give the numerical results on the MARCO performance. We also present the performance comparisons of MARCO with the previously proposed molecular communication models in Section IV. Finally, we discuss concluding remarks in Section V.

\section{Molecular Array-Based Communication}

In Molecular ARray-based COmmunication (MARCO), information is encoded by the transmission order of the molecules as shown in Fig. 1. TN selects a molecule at a time among $n$ distinct types of molecules and emits to the medium. The inter-emitting time of the molecules is assumed to be set to $t_{e}$. Thus, if the first molecule is emitted at time $t$, the second is emitted at time $t+t_{e}$, and, so on. In conventional transmission systems, whose alphabet includes two symbols, i.e., $\{0,1\}$, if a codeword is a sequence of $k$ symbols, there are at most $2^{k}$ different codewords. In general, if the alphabet contains $l$ different symbols, the resulting number of possible codewords is $l^{k}$. As in conventional channels, let us investigate the binary case $^{1}$, that is, $l=2$, and there are only two different molecules, namely $a$ and $b$ to transmit information. With no loss of generality, let us associate the transmission order $a-b$ with bit 0 and $b-a$ with bit 1 . More specifically, 0 is transmitted by first emitting molecule $a$ and then $b$, while 1 is transmitted by first emitting $b$ and then $a$. Hence, the information is encoded with the order of the transmitted molecules.

We assume that the movement of molecules is governed by a Brownian motion [20]. The delay $t$ experienced by any molecule to reach $\mathrm{RN}$ at distance $d$ obeys the following probability density function:

$$
f(t)=\frac{\lambda}{\sqrt{4 \pi t^{3}}} e^{-\frac{\lambda^{2}}{4 t}}, \quad t>0
$$

where $\lambda=\frac{d}{\sqrt{D}}$ and $D$ is the diffusion coefficient of the molecules. The cumulative distribution function associated

\footnotetext{
${ }^{1}$ Throughout the paper, we only investigate the binary case with two distinct molecules. The case of $n$ distinct molecules is out of the scope of this paper.
} 
with the density function $f(t)$, i.e., $F(t)$, can be given as

$$
F(t)=1-\operatorname{Erf}\left(\frac{d}{2 \sqrt{D t}}\right), \quad t>0
$$

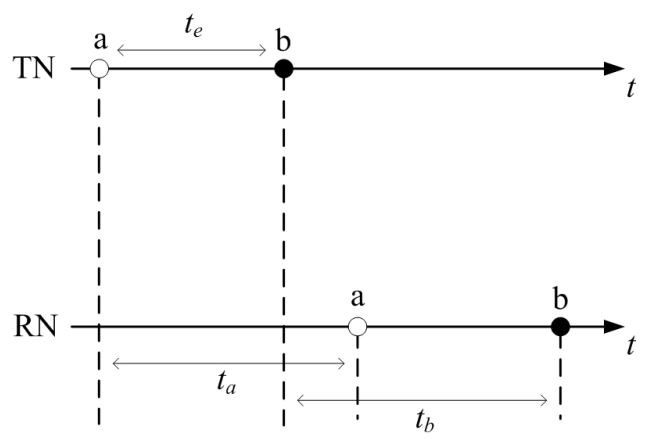

Fig. 2. Transmission and correct reception of two molecules in order $a-b$ which corresponds to the symbol 0 .

Let us now consider the scenario in which $\mathrm{TN}$ sends a symbol 0 using the molecular order $a-b$ to $\mathrm{RN}$ and $\mathrm{RN}$ correctly receives this symbol. The time diagram of this scenario is presented in Fig. 2. The probability of such event can be characterized as

$$
\operatorname{Pr}(0 \mid 0)=\operatorname{Pr}\left(t_{b}>t_{a}-t_{e}\right)= \begin{cases}1, & t_{a}<t_{e} \\ I\left(t_{e}, \lambda\right), & t_{a}>t_{e}\end{cases}
$$

where $\operatorname{Pr}(0 \mid 0)$ denotes the probability that symbol 0 is transmitted by TN and correctly received by RN. $t_{a}$ and $t_{b}$ are the random time delays experienced by molecule $a$ and $b$, respectively. Note that since $t_{a}$ and $t_{b}$ have the same probability distribution given in (1), i.e., $f\left(t_{a}\right)=f\left(t_{b}\right)=f(t)$, the transmission of symbol 0 with the order $a-b$ is statistically equal to the transmission of symbol 1 with the order $b-a$, that is, $\operatorname{Pr}(0 \mid 0)=\operatorname{Pr}(1 \mid 1)$. In (3), $I\left(t_{e}, \lambda\right)$ denotes the probability function that depends on the inter-emission time $t_{e}$ and the ratio $\lambda$. In fact, (3) can be rewritten in a more compact form as

$$
\operatorname{Pr}(0 \mid 0)=1 \times \operatorname{Pr}\left(t_{a}<t_{e}\right)+I\left(t_{e}, \lambda\right) \times\left(1-\operatorname{Pr}\left(t_{a}<t_{e}\right)\right)
$$

Using the probability distribution of $t_{a}$ and $t_{b}$ in (1), the function $I\left(t_{e}, \lambda\right)$ can be derived as

$$
\begin{aligned}
I\left(t_{e}, \lambda\right) & =\int_{t_{e}}^{\infty} \int_{t_{a}-t_{e}}^{\infty} f\left(t_{a}, t_{b}\right) d t_{a} d t_{b} \\
& =\int_{t_{e}}^{\infty} \int_{t_{a}-t_{e}}^{\infty} f\left(t_{a}\right) f\left(t_{b}\right) d t_{a} d t_{b} \\
& =\int_{t_{e}}^{\infty} f\left(t_{a}\right) \operatorname{Erf}\left(\frac{\lambda}{2 \sqrt{t_{a}-t_{e}}}\right) d t_{a}
\end{aligned}
$$

where $f\left(t_{a}, t_{b}\right)$ denotes the joint density function of the random delays $t_{a}$ and $t_{b}$. Since these delays are assumed to be independent, $f\left(t_{a}, t_{b}\right)=f\left(t_{a}\right) f\left(t_{b}\right)$. Furthermore, $\operatorname{Pr}\left(t_{a}<\right.$ $\left.t_{e}\right)$ in (4), i.e., the probability that $t_{a}$ does not exceed the interemission time $t_{e}$, can be derived as

$$
\operatorname{Pr}\left(t_{a}<t_{e}\right)=\int_{0}^{t_{e}} f\left(t_{a}\right) d t_{a}=1-\operatorname{Erf}\left(\frac{\lambda}{2 \sqrt{t_{e}}}\right)
$$

Consequently, substituting (5) and (6) into (4), $\operatorname{Pr}(0 \mid 0)$ can be rewritten in a more explicit form, i.e.,

$$
\begin{array}{r}
\operatorname{Pr}(0 \mid 0)=\left[1-\operatorname{Erf}\left(\frac{\lambda}{2 \sqrt{t_{e}}}\right)\right]+ \\
+\operatorname{Erf}\left(\frac{\lambda}{2 \sqrt{t_{e}}}\right) \int_{t_{e}}^{\infty} f\left(t_{a}\right) \operatorname{Erf}\left(\frac{\lambda}{2 \sqrt{t_{a}-t_{e}}}\right)
\end{array}
$$

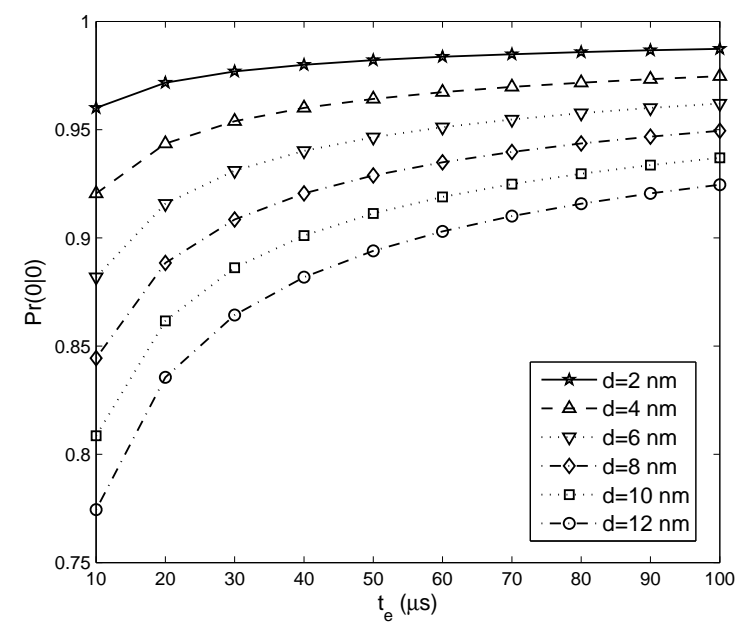

Fig. 3. Probability of correct symbol reception, i.e., $\operatorname{Pr}(0 \mid 0)$, with respect to the inter-emission time $t_{e}$ for the different distances between TN and RN.

The successful transmission probability $\operatorname{Pr}(0 \mid 0)$ in (7) is plotted in Fig. 3 as a function of the inter-emission time $t_{e}$ for different values of the inter-node distance $d$ between TN and $\mathrm{RN}$. The results reveal an intuitively expected behavior such that, as $d$ decreases and $t_{e}$ increases, the probability of successful reception of a symbol increases. In other words, a larger $d$ requires a larger $t_{e}$ to yield a high probability of correct reception. At the same time, the numerical evaluation of $\operatorname{Pr}(0 \mid 0)$ manifests that for sufficiently large $d$, e.g., in the order of ten times larger (like $120 \mathrm{~nm}$ ), $\operatorname{Pr}(0 \mid 0)$ becomes almost independent of the inter-emission time, and flattens around 0.5 . This result could be expected since the interemission time becomes irrelevant and any molecule may arrive first when the distance is so large.

In addition to the case where a single symbol is out-of-order, two consecutive (binary) symbols may also mutually interfere with each other, which causes erroneous transmission. This phenomenon is called as Inter-Symbol Interference (ISI). Here, we assume that a transmitted symbol merely interferes with either previously or next transmitted symbols. Hence, considering the symbol triplet 000 , we derive the ISI probability, i.e., $P_{I S I}$, that the currently transmitted symbol 0 interferes with the previously or next transmitted symbol 0 . The relevant delay variables needed for this derivation can be defined as follows.

\section{A. The Relevant Delay Variables in MARCO Channel}

In the transmission of $000, t_{a}$ and $t_{b}$ denote the random delay of molecules $a$ and $b$ in the first symbol 0 , respectively 


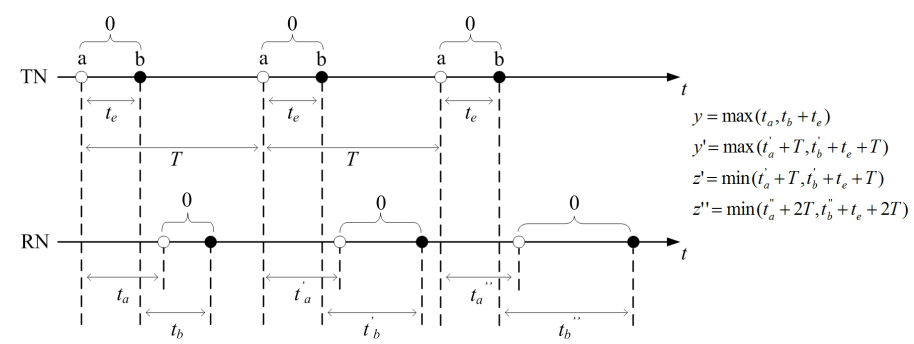

Fig. 4. Time diagram for the transmission and correct reception of three consecutive symbols 000 using the molecular order $a-b, a-b, a-b$.

as shown in Fig. 4. In this figure, $T$ represents the inter-symbol time. The maximum of delay experienced by the molecules $a$ and $b$ can be statistically characterized by the random variable $y$, i.e., $y=\max \left(t_{a}, t_{b}+t_{e}\right)$. Note that since $t_{a}$ and $t_{b}$ are drawn according to the same density and cumulative distribution functions, i.e., $f(t)$ and $F(t)$ given in (1) and (2), respectively, $t_{a}$ and $t_{b}$ are identically distributed and equivalent to the delay of any other molecule of the same or different symbols. Therefore, the density and cumulative distribution functions of the random variable $y$ can also be derived using $f(t)$ and $F(t)$. Due to the fact that $t_{a}$ and $t_{e}+t_{b}$ are mutually independent, the cumulative distribution function of $y$, i.e., $F_{y}(t)$, can be expressed as

$$
F_{y}(t)=F_{t_{a}}(t) F_{t_{e}+t_{b}}(t), \quad t \geq t_{e}
$$

where $F_{t_{e}+t_{b}}(t)$ can be obtained by time-shifting $F_{t_{b}}(t)$ by $t_{e}$, i.e., $F_{t_{e}+t_{b}}(t)=F_{t_{b}}\left(t-t_{e}\right)$. Because $t_{a}$ and $t_{b}$ are statistically equivalent, $F_{t_{a}}(t)$ and $F_{t_{b}}(t)$ are the cumulative distribution functions given in (2), that is, $F_{t_{a}}(t)=F_{t_{b}}(t)=F(t)=$ $1-\operatorname{Erf}\left(\frac{d}{2 \sqrt{D t}}\right)$. Accordingly, (8) can be written as

$$
\begin{aligned}
F_{y}(t) & =F_{t_{a}}(t) F_{t_{e}+t_{b}}(t) \\
& =F_{t_{a}}(t) F_{t_{b}}\left(t-t_{e}\right) \\
& =\left[1-\operatorname{Erf}\left(\frac{d}{2 \sqrt{D t}}\right)\right] \times \\
& \times\left[1-\operatorname{Erf}\left(\frac{d}{2 \sqrt{D\left(t-t_{e}\right)}}\right)\right]
\end{aligned}
$$

By taking the derivative of (8), the probability density function of $y$, i.e., $f_{y}(t)$, can be also given as

$$
f_{y}(t)=f(t) F\left(t-t_{e}\right)+F(t) f\left(t-t_{e}\right), \quad t \geq t_{e}
$$

$F_{y}(t)$ is plotted in Fig. 5 for $d=4 \mathrm{~nm}$ and for different values of the inter-emission time, i.e., $t_{e}$. Note that $t_{e}$ should correspond to a value of the argument $t$ such that the distribution function is sufficiently large, for instance 0.9. In this case, the inter-symbol time is nothing else but the percentile-90 of the corresponding density function. For instance, for an inter-emission time of $20 \mu \mathrm{s}$, the value of $t$ for which the distribution function is around 0.9 is approximately $40 \mu \mathrm{s}$. This would be the inter-symbol time or simply the slot duration. The corresponding transmission rate would be approximately equal to $25 \mathrm{Kbps}$.

In addition to the first symbol 0 , the delay of molecules $a$ and $b$ in the second symbol 0 can be denoted as $t_{a}^{\prime}$ and $t_{b}^{\prime}$ as

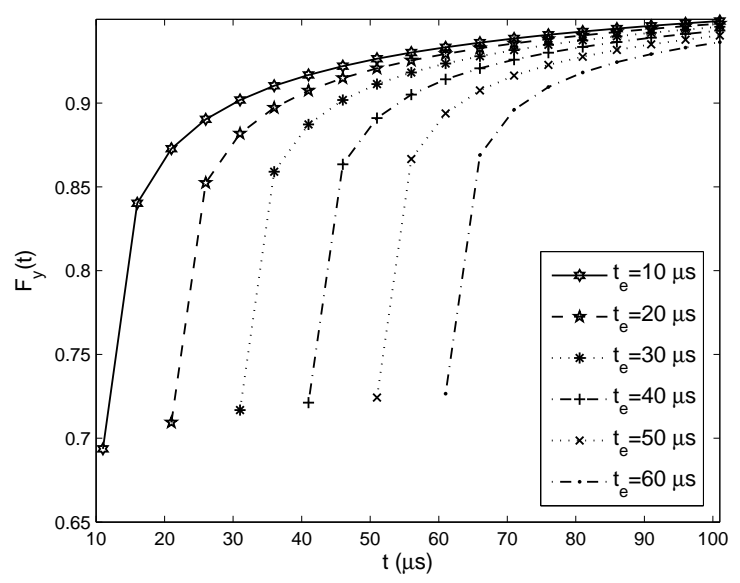

Fig. 5. Distribution function for the evaluation of the inter-symbol time $(d=4 \mathrm{~nm})$.

shown in Fig. 4. Moreover, the minimum and maximum of the delay experienced by the molecules $a$ and $b$ in the second symbol 0 can be also characterized by the random variable $z^{\prime}$ and $y^{\prime}$, respectively, i.e., $z^{\prime}=\min \left(t_{a}^{\prime}+T, t_{b}^{\prime}+t_{e}+T\right)$ and $y^{\prime}=\max \left(t_{a}^{\prime}+T, t_{b}^{\prime}+t_{e}+T\right)$. Note that the second 0 is transmitted after an inter-symbol time $T$, which is clearly observed in Fig. 4. Here, the cumulative distribution function of $z^{\prime}=\min \left(t_{a}^{\prime}+T, t_{b}^{\prime}+t_{e}+T\right)$, i.e., $F_{z^{\prime}}(t)$, can be derived as

$$
\begin{aligned}
F_{z^{\prime}}(t) & =F(t-T)+F\left(t-t_{e}-T\right)- \\
& -F(t-T) F\left(t-t_{e}-T\right), \quad t \geq T
\end{aligned}
$$

By taking the derivative of $F_{z^{\prime}}(t)$, the density function of $z^{\prime}$, i.e., $f_{z^{\prime}}(t)$, can be also found as

$$
\begin{aligned}
f_{z^{\prime}}(t) & =f(t-T)\left[1-F\left(t-t_{e}-T\right)\right]+ \\
& +f\left(t-t_{e}-T\right)[1-F(t-T)], \quad t \geq T
\end{aligned}
$$

Similarly, the time delays experienced by the molecules $a$ and $b$ in the third symbol 0 are denoted as $t_{a}^{\prime \prime}$ and $t_{b}^{\prime \prime}$, respectively as seen in Fig. 4. $z^{\prime \prime}$ are also the random variable that characterizes the minimum of the delay experienced by the molecules $a$ and $b$ in the third symbol 0 , i.e., $z^{\prime \prime}=$ $\min \left(t_{a}^{\prime \prime}+2 T, t_{b}^{\prime \prime}+t_{e}+2 T\right)$. Here, note also that the third 0 is transmitted after two inter-symbol times $(2 T)$ as shown in Fig. 4. The density function of $z^{\prime \prime}$, i.e., $f_{z^{\prime \prime}}(t)$, can be also obtained by replacing $T$ in $f_{z^{\prime}}(t)$ in (12) with $2 T$, i.e.,

$$
\begin{aligned}
& f_{z^{\prime \prime}}(t)=f(t-2 T)\left[1-F\left(t-t_{e}-2 T\right)\right]+ \\
& +f\left(t-t_{e}-2 T\right)[1-F(t-2 T)], \quad t \geq 2 T
\end{aligned}
$$

Next, we derive the ISI probability in the MARCO channel using the delay variables defined above. 


\section{B. Probability of ISI in MARCO Channel}

The probability of ISI, i.e., $P_{I S I}$, can be first formulated as

$$
\begin{aligned}
P_{I S I} & =1-P_{\text {NoISI }}=1-\operatorname{Pr}\left(\left(z^{\prime}>y\right) \cap\left(y^{\prime}<z\right)\right) \\
& =1-\operatorname{Pr}\left(y^{\prime}<z^{\prime \prime} \mid z^{\prime}>y\right) \operatorname{Pr}\left(z^{\prime}>y\right)
\end{aligned}
$$

where $P_{N o I S I}$ denotes the probability that a transmitted symbol does not interfere with any other symbol. (14) highlights the fact that $y^{\prime}$ and $z^{\prime}$ are not independent variables, because they are evaluated from the same pair $\left(t_{a}^{\prime}, t_{b}^{\prime}\right)$. Hence, the conditioned probability $\operatorname{Pr}\left(y^{\prime}<z^{\prime \prime} \mid z^{\prime}>y\right)$ in (14) can be derived as

$$
\begin{aligned}
\operatorname{Pr}\left(y^{\prime}<z^{\prime \prime} \mid z^{\prime}>y\right) & =\operatorname{Pr}\left(t_{a}^{\prime}+T<z^{\prime \prime} \mid t_{b}^{\prime}+t_{e}+T>y\right) \\
& \times \operatorname{Pr}\left(t_{a}^{\prime}+T>t_{b}^{\prime}+t_{e}+T\right) \\
& +\operatorname{Pr}\left(t_{b}^{\prime}+t_{e}+T<z^{\prime \prime} \mid t_{a}^{\prime}+T>y\right) \\
& \times \operatorname{Pr}\left(t_{b}^{\prime}+t_{e}+T>t_{a}^{\prime}+T\right)
\end{aligned}
$$

Here, the two possible cases are considered, i.e., either $y^{\prime}=$ $t_{a}^{\prime}+T$ and $z^{\prime}=t_{b}^{\prime}+t_{e}+T$ or vice versa, that is, $y^{\prime}=t_{b}^{\prime}+t_{e}+T$ and $z^{\prime}=t_{a}^{\prime}+T$. Due to the independence of $t_{a}^{\prime}$ and $t_{b}^{\prime}$, (15) can be rewritten as

$$
\begin{array}{r}
\operatorname{Pr}\left(y^{\prime}<z^{\prime \prime} \mid z^{\prime}>y\right)=\operatorname{Pr}\left(t_{a}^{\prime}<z^{\prime \prime}-T\right) \operatorname{Pr}\left(t_{a}^{\prime}>t_{b}^{\prime}+t_{e}\right) \\
+\operatorname{Pr}\left(t_{b}^{\prime}<z^{\prime \prime}-t_{e}-T\right) \operatorname{Pr}\left(t_{a}^{\prime}<t_{b}^{\prime}+t_{e}\right)
\end{array}
$$

However, we know that $\operatorname{Pr}\left(t_{a}^{\prime}<t_{b}^{\prime}+t_{e}\right)=\operatorname{Pr}(0 \mid 0)$ and obviously $\operatorname{Pr}\left(t_{a}^{\prime}>t_{b}^{\prime}+t_{e}\right)=1-\operatorname{Pr}(0 \mid 0)$, where $\operatorname{Pr}(0 \mid 0)$ is already known as given in (7). Accordingly, (16) can be rewritten as

$$
\begin{aligned}
\operatorname{Pr}\left(y^{\prime}<z^{\prime \prime} \mid z^{\prime}>y\right) & =\operatorname{Pr}\left(t_{a}^{\prime}<z^{\prime \prime}-T\right)[1-\operatorname{Pr}(0 \mid 0)]+ \\
& +\operatorname{Pr}\left(t_{b}^{\prime}<z^{\prime \prime}-t_{e}-T\right) \operatorname{Pr}(0 \mid 0)(17)
\end{aligned}
$$

In order to derive $\operatorname{Pr}\left(t_{a}^{\prime}<z^{\prime \prime}-T\right)$ in (17), we notice that $\operatorname{Pr}\left(t_{a}^{\prime}<z^{\prime \prime}-T\right)=\operatorname{Pr}\left(t_{a}^{\prime}-z^{\prime \prime}<-T\right)$. Let us now define $r=-z^{\prime \prime}$. Using $f_{z^{\prime \prime}}(t)$ derived in (13), the density function of the random variable $r$, i.e., $f_{r}(t)$, can be easily written as

$$
\begin{gathered}
f_{r}(t)=f(-t-2 T)\left[1-F\left(-t-t_{e}-2 T\right)\right]+ \\
+f\left(-t-t_{e}-2 T\right)[1-F(-t-2 T)], \quad t \leq-2 T
\end{gathered}
$$

Since $t_{a}$ and $r$ are independent, the density function of $t_{a}+r$, i.e., $f_{t_{a}+r}(t)$, can be obtained by

$$
f_{t_{a}+r}(t)=\int_{\bar{u}}^{\infty} f(u) f_{r}(t-u) d u
$$

where $\bar{u}=\max (0, t+2 T)$. Hence, using (19), $\operatorname{Pr}\left(t_{a}^{\prime}<z^{\prime \prime}-\right.$ $T)$ can be computed as

$$
\begin{aligned}
\operatorname{Pr}\left(t_{a}^{\prime}<z^{\prime \prime}-T\right) & =\operatorname{Pr}\left(t_{a}^{\prime}+r<-T\right) \\
& =\int_{-\infty}^{-T} f_{t_{a}+r}(t) d t
\end{aligned}
$$

Similarly, $\operatorname{Pr}\left(t_{a}^{\prime}<z^{\prime \prime}-t_{e}-T\right)$ in (17) can be also computed as

$$
\operatorname{Pr}\left(t_{a}^{\prime}<z^{\prime \prime}-t_{e}-T\right)=\int_{-\infty}^{-t_{e}-T} f_{t_{a}+r}(t) d t
$$

Here, for the ease of illustration, let us introduce $q(\alpha)=$ $\int_{\text {as }}^{-\alpha} f_{t_{a}+r}(t) d t$. Thus, $\operatorname{Pr}\left(y^{\prime}<z^{\prime \prime} \mid z^{\prime}>y\right)$ can be written $\operatorname{Pr}\left(y^{\prime}<z^{\prime \prime} \mid z^{\prime}>y\right)=q(T)(1-\operatorname{Pr}(0 \mid 0))+q\left(t_{e}+T\right) \operatorname{Pr}(0 \mid 0)$

Furthermore, substituting (22) into (14), $P_{I S I}$ can be expressed as

$P_{I S I}=1-\left[q(T)[1-\operatorname{Pr}(0 \mid 0)]+q\left(t_{e}+T\right) \operatorname{Pr}(0 \mid 0)\right] \operatorname{Pr}\left(z^{\prime}>y\right)$

In (23), $\operatorname{Pr}(0 \mid 0), q(T)$, and $q\left(t_{e}+T\right)$ are known as they are derived above. However, $\operatorname{Pr}\left(z^{\prime}>y\right)$ should be derived in order to obtain an exact expression for $P_{I S I}$. To this end, we define another random variable $s=-y$ such that $\operatorname{Pr}\left(z^{\prime}<y\right)$ can be calculated as

$$
\operatorname{Pr}\left(z^{\prime}<y\right)=\operatorname{Pr}\left(z^{\prime}+(-y)<0\right)=\operatorname{Pr}\left(z^{\prime}+s<0\right)
$$

Using $f_{y}(t)$ in (10), the density function of $s$, i.e., $f_{s}(t)$, can be expressed as

$$
\begin{aligned}
f_{s}(t)= & f_{y}(-t)=f(-t) F\left(-t-t_{e}\right)+ \\
& +F(-t) f\left(-t-t_{e}\right), \quad t \leq-t_{e} .
\end{aligned}
$$

Since $z^{\prime}$ and $s$ are two independent random variables, the density function $f_{z^{\prime}+s}(t)$ can be obtained by

$$
\begin{aligned}
f_{z^{\prime}+s}(t) & =\int_{-\infty}^{\infty} f_{z^{\prime}}(u) f_{s}(t-u) d u \\
& =\int_{T}^{\infty} f_{z^{\prime}}(u) f_{s}(t-u) d u \\
& =\int_{\bar{o}}^{\infty} f_{z^{\prime}}(u) f_{s}(t-u) d u
\end{aligned}
$$

where $\bar{o}=\max \left(t+t_{e}, T\right)$ and $f_{z^{\prime}}(t)$ is the density function of random variable $z^{\prime}=\min \left(t_{a}^{\prime}+T, t_{b}^{\prime}+t_{e}+T\right)$ and given in (12). Hence, using $f_{z^{\prime}+s}(t), \operatorname{Pr}\left(z^{\prime}<y\right)$ can be evaluated as

$$
\operatorname{Pr}\left(z^{\prime}<y\right)=\operatorname{Pr}\left(z^{\prime}+s<0\right)=\int_{-\infty}^{0} f_{z^{\prime}+s}(t) d t
$$

Again, for the ease of illustration, let us introduce another variable $\tilde{p}$ as $\tilde{p}=\operatorname{Pr}\left(z^{\prime}<y\right)=\int_{-\infty}^{0} f_{z^{\prime}+s}(t) d t$. Due to $\operatorname{Pr}\left(z^{\prime}>y\right)=1-\operatorname{Pr}\left(z^{\prime}<y\right)$, by using $\widetilde{p}=\operatorname{Pr}\left(z^{\prime}<y\right)$, $\operatorname{Pr}\left(z^{\prime}>y\right)$ can be expressed as

$$
\operatorname{Pr}\left(z^{\prime}>y\right)=1-\operatorname{Pr}\left(z^{\prime}<y\right)=1-\widetilde{p}
$$

Finally, by substituting $\operatorname{Pr}\left(z^{\prime}>y\right)=1-\widetilde{p}$ into (23), a definite expression for $P_{I S I}$ can be given as

$P_{I S I}=1-\left[q(T)[1-\operatorname{Pr}(0 \mid 0)]+q\left(t_{e}+T\right) \operatorname{Pr}(0 \mid 0)\right](1-\widetilde{p})$

Substituting $\operatorname{Pr}(0 \mid 0), q(t), q\left(t_{e}+T\right)$, and $\widetilde{p}$ into (29), $P_{I S I}$ can be also rewritten as where $f_{z^{\prime}}(t), f_{r}(t)$, and $f_{s}(t)$ are given in (12), (18), and (25), respectively. Furthermore, $\bar{u}$ and $\bar{o}$ are given as $\bar{u}=\max (0, t+2 T)$ and $\bar{o}=\max \left(t+t_{e}, T\right)$. Next, we also derive the error probability on which the communication rate for the MARCO channel is based. 


$$
\begin{aligned}
P_{I S I} & =1-\left[\left(\int_{-\infty}^{-T} \int_{\bar{u}}^{\infty} f(u) f_{r}(t-u) d u d t\right)\left[1-\left(\operatorname{Erfc}\left(\frac{\lambda}{2 \sqrt{t_{e}}}\right)+\operatorname{Erf}\left(\frac{\lambda}{2 \sqrt{t_{e}}}\right) \int_{t_{e}}^{\infty} f\left(t_{a}\right) \operatorname{Erf}\left(\frac{\lambda}{2 \sqrt{t_{a}-t_{e}}}\right) d t_{a}\right)\right]+\right. \\
& \left.+\left(\int_{-\infty}^{-\left(t_{e}+T\right)} \int_{\bar{u}}^{\infty} f(u) f_{r}(t-u) d u d t\right)\left(\operatorname{Erfc}\left(\frac{\lambda}{2 \sqrt{t_{e}}}\right)+\operatorname{Erf}\left(\frac{\lambda}{2 \sqrt{t_{e}}}\right) \int_{t_{e}}^{\infty} f\left(t_{a}\right) \operatorname{Erf}\left(\frac{\lambda}{2 \sqrt{t_{a}-t_{e}}}\right) d t_{a}\right)\right] \times \\
& \times\left(1-\int_{-\infty}^{0} \int_{\bar{o}}^{\infty} f_{z^{\prime}}(u) f_{s}(t-u) d u d t\right)
\end{aligned}
$$

\section{Derivation of Error Probability in MARCO Channel}

As observed in (29), $P_{I S I}$ is statistically dependent on the successful transmission probability $\operatorname{Pr}(0 \mid 0)^{2}$. Hence, it cannot be possible to obtain the error probability in the MARCO channel by simply factorizing $P_{I S I}$ and $(1-\operatorname{Pr}(0 \mid 0))$. In fact, the probability of error, i.e., $P_{e}$, can be formulated as

$$
P_{e}=1-P_{N o I S I \mid(0 \mid 0)} \operatorname{Pr}(0 \mid 0)
$$

where $P_{N o I S I \mid(0 \mid 0)}$ is the probability that a symbol does not interfere given the fact that it is correctly transmitted. Then, according to Fig. 4, $P_{N o I S I \mid(0 \mid 0)}$ can be derived as

$$
\begin{aligned}
P_{\text {NoISI } \mid(0 \mid 0)} & =\operatorname{Pr}\left(\left(z^{\prime}>y\right) \cap\left(y^{\prime}<z^{\prime \prime}\right) \mid(0 \mid 0)\right) \\
& =\operatorname{Pr}\left(y^{\prime}<z^{\prime \prime} \mid z^{\prime}>y,(0 \mid 0)\right) \times \\
& \times \operatorname{Pr}\left(z^{\prime}>y \mid(0 \mid 0)\right)
\end{aligned}
$$

where $\operatorname{Pr}\left(y^{\prime}<z^{\prime \prime} \mid z^{\prime}>y,(0 \mid 0)\right)$ in (32) can be expressed as $\operatorname{Pr}\left(y^{\prime}<z^{\prime \prime} \mid z^{\prime}>y,(0 \mid 0)\right)=\operatorname{Pr}\left(t_{b}^{\prime}+t_{e}+T<z^{\prime \prime} \mid t_{a}^{\prime}+T>y\right)$

More specifically,

$$
\begin{aligned}
\operatorname{Pr}\left(y^{\prime}<z^{\prime \prime} \mid z^{\prime}>y,(0 \mid 0)\right) & =\operatorname{Pr}\left(t_{b}^{\prime}<z^{\prime \prime}-t_{e}-T\right) \\
& =q\left(t_{e}+T\right)
\end{aligned}
$$

where we note that the result $\operatorname{Pr}\left(t_{b}^{\prime}<z^{\prime \prime}-t_{e}-T\right)=q\left(t_{e}+\right.$ $T$ ) has already been derived in (21). Moreover, in order to complete the derivation of $P_{N o I S I \mid(0 \mid 0)}, \operatorname{Pr}\left(z^{\prime}>y \mid(0 \mid 0)\right)$ in (32) can be given as

$$
\begin{aligned}
\operatorname{Pr}\left(z^{\prime}>y \mid(0 \mid 0)\right) & =\operatorname{Pr}\left(t_{a}^{\prime}+T>y\right) \\
& =\operatorname{Pr}\left(t_{a}^{\prime}-y>-T\right)
\end{aligned}
$$

As in (24), let us again define the random variable $s=-y$ and rewrite (34) as

$$
\begin{aligned}
\operatorname{Pr}\left(z^{\prime}>y \mid(0 \mid 0)\right) & =\operatorname{Pr}\left(t_{a}^{\prime}+(-y)>-T\right) \\
& =\operatorname{Pr}\left(t_{a}^{\prime}+s>-T\right)
\end{aligned}
$$

Note that the density function of $s$, i.e., $f_{s}(t)$, is already derived in (25). Since $t_{a}^{\prime}$ and $s$ are assumed to be statistically independent, the density function of $t_{a}^{\prime}+s$, i.e., $f_{t_{a}+s}(t)$, is found as

$$
\begin{aligned}
f_{t_{a}+s}(t) & =\int_{-\infty}^{+\infty} f(u) f_{s}(t-u) d u \\
& =\int_{0}^{+\infty} f(u) f_{s}(t-u) d u \\
& =\int_{\bar{h}}^{+\infty} f(u) f_{s}(t-u) d u
\end{aligned}
$$

\footnotetext{
${ }^{2}$ However, this is not the case for very small $t_{e}$, and therefore, $q(T) \cong$ $q\left(t_{e}+T\right)$ and $\operatorname{Pr}(0 \mid 0)$ disappears from the $P_{I S I}$ expression.
}

where $\bar{h}=\max \left(0, t+t_{e}\right)$ and we notice that $f_{t_{a}+s}(t)=f_{t_{a}^{\prime}+s}(t)$ since $t_{a}$ and $t_{a}^{\prime}$ are statistically equal to each other. Using $f_{t_{a}+s}(t), \operatorname{Pr}\left(z^{\prime}>y \mid(0 \mid 0)\right)$ in (32) can be developed as follows

$$
\begin{aligned}
\operatorname{Pr}\left(z^{\prime}>y \mid(0 \mid 0)\right) & =\operatorname{Pr}\left(t_{a}^{\prime}+s>-T\right) \\
& =1-F_{t_{a}+s}(-T) \\
& =1-\int_{-\infty}^{-T} f_{t_{a}+s}(t) d t
\end{aligned}
$$

Here, assuming that $\widetilde{q}(\beta)=\int_{-\infty}^{-\beta} f_{t_{a}+s}(t) d t, \operatorname{Pr}\left(z^{\prime}>\right.$ $y \mid(0 \mid 0))$ can be rewritten as

$$
\operatorname{Pr}\left(z^{\prime}>y \mid(0 \mid 0)\right)=(1-\widetilde{q}(T))
$$

Consequently, substituting (33) and (38) into (32), $P_{N o I S I \mid(0 \mid 0)}$ can be written as

$$
P_{N o I S I \mid(0 \mid 0)}=\operatorname{Pr}\left(y^{\prime}<z^{\prime \prime} \mid z^{\prime}>y,(0 \mid 0)\right) \operatorname{Pr}\left(z^{\prime}>y \mid(0 \mid 0)\right)
$$

That is,

$$
P_{N o I S I \mid(0 \mid 0)}=q\left(t_{e}+T\right)(1-\widetilde{q}(T))
$$

Finally, substituting $P_{N o I S I \mid(0 \mid 0)}$ given in (39) into (31), the error probability, i.e., $P_{e}$, can be given as

$$
\begin{aligned}
P_{e} & =1-P_{N o I S I \mid(0 \mid 0)} \operatorname{Pr}(0 \mid 0) \\
& =1-q\left(t_{e}+T\right)(1-\widetilde{q}(T)) \operatorname{Pr}(0 \mid 0)
\end{aligned}
$$

where $q($.$) are \widetilde{q}($.$) are already known since they are defined$ in Section II-B and II-C, respectively. $\operatorname{Pr}(0 \mid 0)$ is expressed in (7). Next, using $P_{e}$ in (40), we explore an achievable communication rate for MARCO.

\section{Rate of Molecular Array-Based Communication}

Here, we derive a simple achievable rate expression of the MARCO channel in which TN transmits either 0 or 1 and $\mathrm{RN}$ either correctly receives the information bit with the probability $\left(1-P_{e}\right)$ or incorrectly receives the bit with the probability $P_{e}$. Therefore, an achievable rate of the MARCO channel in bits per transmission, i.e., $C$, can be characterized as $C=\left(1-P_{e}\right)$. Hence, by substituting the error probability $P_{e}$ in (40), the communication rate, i.e., $C=\left(1-P_{e}\right)$, can be expressed as

$$
C=q\left(t_{e}+T\right)(1-\widetilde{q}(T)) \operatorname{Pr}(0 \mid 0)
$$

Substituting $q\left(t_{e}+T\right), \widetilde{q}(T)$, and $\operatorname{Pr}(0 \mid 0)$ into (41), $C$ can be also rewritten as given in (42). In (42), $f_{r}(t)$ and $f_{s}(t)$ 


$$
\begin{aligned}
C & =\left[\int_{-\infty}^{-\left(t_{e}+T\right)} \int_{\bar{u}}^{\infty} f(u) f_{r}(t-u) d u d t\right] \times\left[1-\int_{-\infty}^{-T} \int_{\bar{h}}^{+\infty} f(u) f_{s}(t-u) d u d t\right] \times \\
& \times\left[\operatorname{Erfc}\left(\frac{\lambda}{2 \sqrt{t_{e}}}\right)+\operatorname{Erf}\left(\frac{\lambda}{2 \sqrt{t_{e}}}\right) \int_{t_{e}}^{\infty} f\left(t_{a}\right) \operatorname{Erf}\left(\frac{\lambda}{2 \sqrt{t_{a}-t_{e}}}\right) d t_{a}\right]
\end{aligned}
$$

are given in (18) and (25), respectively. Furthermore, $\bar{u}$ and $\bar{h}$ are given as $\bar{u}=\max (0, t+2 T)$ and $\bar{h}=\max \left(0, t+t_{e}\right)$, respectively. $\bar{u}$ and $\bar{h}$ are derived from the domains of functions $f, f_{r}$ and $f_{s}$ or, to be more specific, from the intersection of these domains: $f$ and $f_{r}$ in the case of $\bar{u}$ and $f$ and $f_{s}$ in the case of $\bar{h}$. The maximization of $C$ provides the maximum number of symbols that can be successfully transmitted per inter-symbol time $T$. Thus, the rate of MARCO in bits per second (bps) can be given as $\frac{C}{T}(b p s)$.

\section{NUMERICAL RESULtS}

In this section, we present the numerical analysis for the performance of MARCO by using the ISI probability $P_{I S I}$, error probability $P_{e}$, and the communication rate $C$ given in (29), (40) and (42), respectively. The performance of MARCO is quantified by the numerical evaluation of these expressions. We first show the effect of inter-emission time and intersymbol time on the MARCO performance. Then, we show how inter-emission time and inter-node distance between TN and RN affect the performance. Finally, we also present the numerical results to demonstrate the effect of diffusion coefficient on the MARCO performance. In the analysis, the interemission time and inter-symbol time are changed within the intervals $t_{e}=2-8 \mu \mathrm{s}$ and $T=10-25 \mu \mathrm{s}$, respectively. The inter-node distance is selected from the interval $d=1-10 \mathrm{~nm}$ and the diffusion coefficient is changed within the interval $D=25-100 \mu \mathrm{m}^{2} / \mathrm{s}$. The numerical computation of $P_{I S I}$, $P_{e}$, and $C$ are performed using Mathematica.

\section{A. Effects of Inter-Emission Time and Inter-Symbol Time}

In Fig. 6(a), ISI probability, $P_{I S I}$, is shown with respect to the changing inter-emission time $t_{e}$ and inter-symbol time $T$. Note that since the inter-emission time $t_{e}$ must be less than the inter-symbol time $T, t_{e}$ is increased at most up to the selected $T$ values. As observed in Fig. 6(a), $P_{I S I}$ can be reduced by increasing $T$, which can be easily verified by visualizing consecutively transmitted symbols. More specifically, as the inter-symbol time increases, it is apparent that the likelihood of inter-symbol interference decreases. Moreover, while $t_{e}$ increases up to some critical values, $P_{I S I}$ first decreases. This result stems from the statistical dependence between $P_{I S I}$ and the successful transmission probability $\operatorname{Pr}(0 \mid 0)$. While $t_{e}$ increases, $\operatorname{Pr}(0 \mid 0)$ can be improved as previously shown in Fig. 3 and this reduces $P_{I S I}$. However, as $t_{e}$ is further increased, $P_{I S I}$ starts to increase because the difference between $t_{e}$ and $T$ becomes too small. That is, after some critical values of $t_{e}$, as the difference between $T$ and $t_{e}$ decreases, almost the all erroneous transmissions, coming from the probability $(1-\operatorname{Pr}(0 \mid 0))$, results in an ISI error and $P_{I S I}$ increases.
Hence, we can conclude that, as the difference between $T$ and $t_{e}$ decreases below some critical values, $P_{I S I}$ cannot be further reduced by increasing $t_{e}$ and starts to increase with $t_{e}$.

Using the same setting in Fig. 6(a), the communication rate of the MARCO channel, i.e., $C / T$ in $b p s$, is shown in Fig. 6(b) for varying $t_{e}$ and $T$ values. In fact, $C$ is given as $C=$ $\left(1-P_{e}\right)$, where $P_{e}$ is the error probability in (40). Therefore, the communication rate $C / T$ is a function of $P_{e}$ and follows the same characteristics with $\left(1-P_{e}\right)$ for a specific $T$. On the other hand, $T$ values in the denominator of $C / T$ suppress the effect of this increase, and $T$ completely controls the rate in a way that the rate can be enhanced as $T$ decreases. As $t_{e}$ increases, $P_{e}$ decreases and $C / T$ can be improved. However, $t_{e}$ gets closer to $T, P_{e}$ and $P_{I S I}$ start to increase and $C / T$ reduces. Moreover, it is revealed that MARCO can provide significantly high communication rate, i.e., up to 45 (Kbps) for the setting in Fig. 6(b).

\section{B. Effects of Inter-Symbol Time and Inter-Node Distance}

In Fig. 7(a), $P_{I S I}$ is shown for varying values of $T$ and $d$. $P_{I S I}$ increases with $d$ due to the fact that longer movement of the molecules causes higher likelihood of ISI. As previously observed in Fig. 6(a), it is also clearly observed in Fig. 7(a) that $P_{I S I}$ decreases with increasing $T$. Here, we can conclude that larger inter-node distance, $d$, requires larger inter-symbol time in order to provide smaller $P_{I S I}$.

Using the same setting in Fig. 7(a), the rate of MARCO, i.e., $C / T$, is shown in Fig. 7(b). The rate decreases as $T$ and $d$ increase. In Fig. 7(b), it is also observed that MARCO can provide very high communication rate, i.e., up to approximately 275 Kbps.

\section{Effects of Inter-Emission Time and Diffusion Coefficient}

In Fig. 8(a), $P_{I S I}$ is depicted for different $t_{e}$ and diffusion coefficient $D$ values. The effect of $t_{e}$ on $P_{I S I}$ is clearly similar to the case in Fig. 6(a) such that as $t_{e}$ increases, $P_{I S I}$ decreases. However, $P_{I S I}$ starts to increase as $t_{e}$ gets closer to $T$. On the other hand, $P_{I S I}$ can be also reduced when the diffusion coefficient $D$ of the molecules is high because higher $D$ decreases the expected value of random delay experienced by each molecule and random delay differences among the molecules. The rate of the MARCO channel, i.e., $C / T$, is shown with varying $t_{e}$ for different values of $D$ in Fig. 8(b). Since $C=\left(1-P_{e}\right)$ and the error probability $P_{e}$ also has similar characteristics with respect to $P_{I S I}$, the rate easily follows a trend opposite to $P_{I S I}$. 


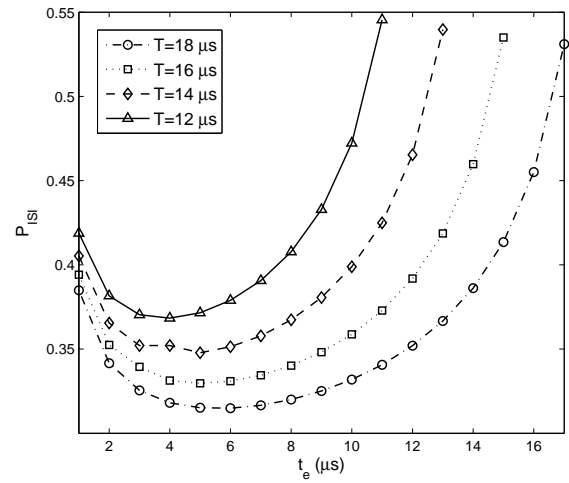

(a)

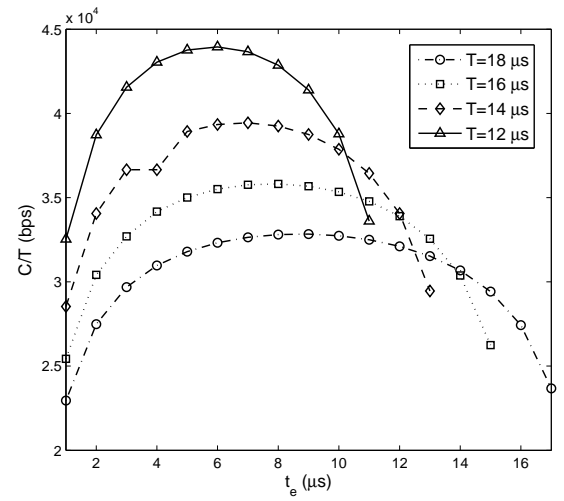

(b)

Fig. 6. (a) The ISI probability values are shown for varying inter-emission time $t_{e}$ and inter-symbol time $T$ values. (b) MARCO communication rate $\frac{C}{T}$ is shown with inter-emission time $t_{e}$ according to the different inter-symbol time $T$ values.

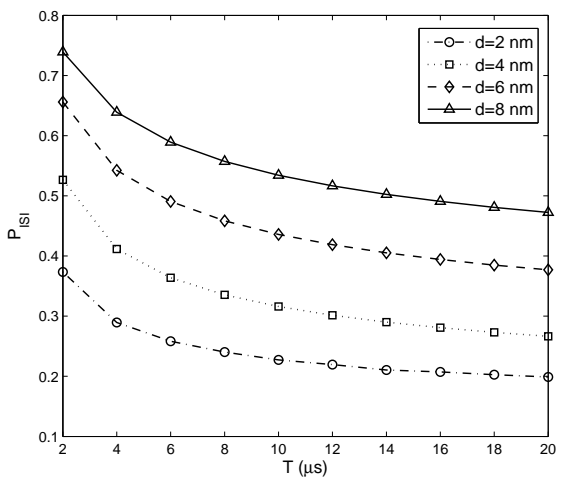

(a)

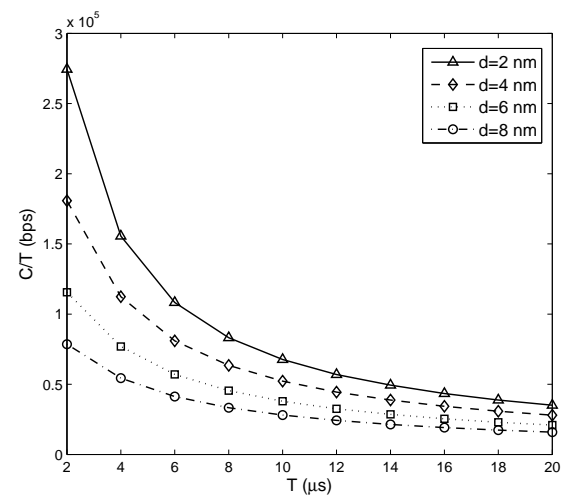

(b)

Fig. 7. (a) $P_{I S I}$ is shown for varying $T$ according to the different inter-node distance $d$ values. (b) $\frac{C}{T}$ is shown for varying $T$ according to the different $d$ values.

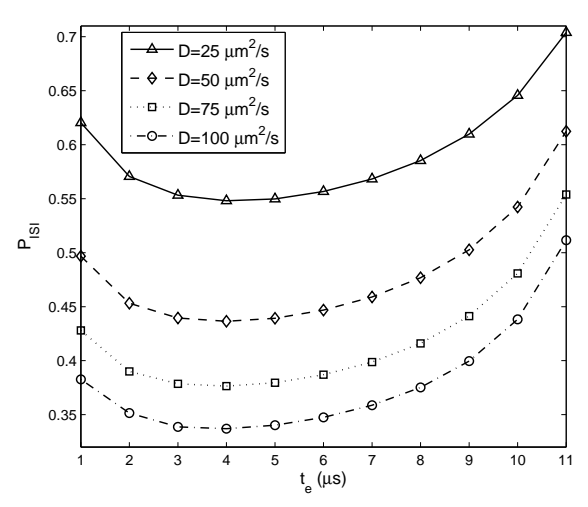

(a)

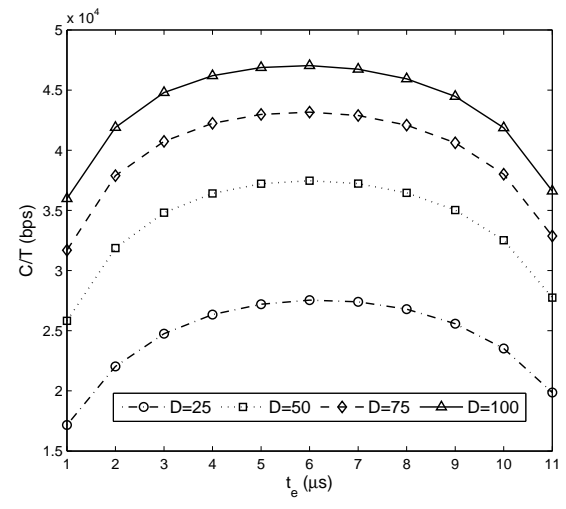

(b)

Fig. 8. (a) $P_{I S I}$ is shown for varying $t_{e}$ according to the different diffusion coefficient $D$ values. (b) $\frac{C}{T}$ is shown for varying $t_{e}$ according to the different $D$ values.

\section{Performance Comparison With Existing Molecular COMMUnication Models}

In this section, we present the analytical performance comparisons of MARCO with the previously proposed molecular communication models. Up to now, molecular communication channel has been considered as either concentration channel or timing channel. In the concentration channel, TN transmits a single or a number of molecules at the beginning of a time slot in order to deliver logic bit 1 and transmits no molecule to $\mathrm{RN}$ to deliver logic 0 during a slot duration. In the existing literature, such a concentration channel is widely investigated in [15], [21], [22], [23]. In the timing channel, random delay of the molecular arrival at the receiver side is considered 


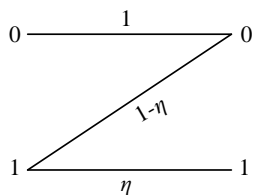

(a)

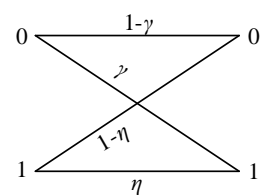

(b)

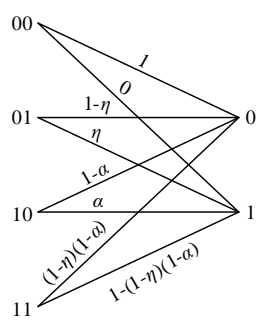

(c)

Fig. 9. (a) Z-channel approach to molecular communication. (b) Binary channel approach. (c) 4-input 2-output memoryless channel approach.

TABLE I

Rate Comparison With Respect To Varying Values of Diffusion Coefficient $\left(\mathrm{D}, \mu \mathrm{m}^{2} / s\right)$

\begin{tabular}{|c|c|c|c|c|c|c|c|c|c|c|}
\hline Diffusion Coefficient & $\mathrm{D}=100$ & $\mathrm{D}=200$ & $\mathrm{D}=300$ & $\mathrm{D}=400$ & $\mathrm{D}=500$ & $\mathrm{D}=600$ & $D=700$ & $\mathrm{D}=800$ & $D=900$ & $D=1000$ \\
\hline Z-channel & $1.1 \times 10^{-41}$ & $8.0 \times 10^{-20}$ & $2.8 \times 10^{-12}$ & $8.1 \times 10^{-9}$ & $1.3 \times 10^{-6}$ & $0.4 \times 10^{-4}$ & $0.4 \times 10^{-3}$ & $0.3 \times 10^{-2}$ & 0.0128 & 0.0411 \\
\hline Binary Channel & $5.7 \times 10^{-20}$ & $5.7 \times 10^{-9}$ & $0.2 \times 10^{-4}$ & $0.2 \times 10^{-2}$ & 0.0290 & 0.1666 & 0.5828 & 1.4895 & 3.0765 & 5.4599 \\
\hline 4-input 2-output Ch. & $1.6 \times 10^{-12}$ & $4.0 \times 10^{-9}$ & $0.2 \times 10^{-4}$ & 0.0015 & 0.0205 & 0.1181 & 0.8279 & 1.0727 & 2.2498 & 4.0743 \\
\hline MARCO Channel & 557.991 & 560.415 & 562.882 & 192.847 & 154.415 & 299.651 & 279.700 & 264.248 & 184.602 & 244.322 \\
\hline
\end{tabular}

TABLE II

Rate Comparison With Respect To Varying Values of Slot and InTER-Symbol Time ( $\tau$ AND $T$ )

\begin{tabular}{l|l|l|l|l}
\hline \hline Slot Time & $\tau=1 \times 10^{-4}$ & $\tau=2 \times 10^{-5}$ & $\tau=1 \times 10^{-5}$ & $\tau=2 \times 10^{-6}$ \\
\hline \hline Z-channel & 0.0411 & $4.0 \times 10^{-19}$ & $1.1 \times 10^{-40}$ & $4.7 \times 10^{-214}$ \\
\hline Binary Channel & 5.4599 & $2.8 \times 10^{-8}$ & $5.7 \times 10^{-19}$ & $1.7 \times 10^{-105}$ \\
\hline 4-input 2-output Ch. & 4.0743 & $2.0 \times 10^{-9}$ & $3.2 \times 10^{-18}$ & $1.3 \times 10^{-101}$ \\
\hline \hline Inter-Symbol Time & $T=1 \times 10^{-4}$ & $T=2 \times 10^{-5}$ & $T=1 \times 10^{-5}$ & $T=2 \times 10^{-6}$ \\
\hline MARCO Channel & 244.322 & 1168.56 & 2324.09 & 11568.50 \\
\hline
\end{tabular}

as a channel noise and the transmission time is the channel input [24]. However, the conditional probability of channel output, given the channel input is analytically intractable in the timing channel. Therefore, an approximate discrete time model in which the information carrying molecules are released at the end of the fixed-duration time slots, is proposed in [24]. This discrete time model renders the timing channel strictly synchronization-dependent and similar to the concentration channel.

In the literature, there are three main modeling approaches to the previously proposed molecular communication models, i.e., Z-channel [23], binary channel [15], [21], [22], [24], and 4-input 2-output discrete memoryless channel approaches [23]. In the Appendix, these approaches are briefly introduced and a rate expression is given for each of them for the performance comparisons with MARCO. Here, we numerically compare the communication rates of MARCO, Z-, binary, and 4-input 2-output channel approaches. The molecular communication rate of Z-, binary, and 4-input 2-output channel models are numerically computed via Mathematica. Apart from the results given in Section III, here, the inter-node distance $d$ is selected on the scale of $\mu$ meters, that is, $d$ is changed within the interval $d=1-10 \mu \mathrm{m}$. Therefore, this section also shows the relatively longer range performance of MARCO. However, we note that these results may vary from the short-range performance of MARCO presented in Section III. The diffusion coefficient is changed from $D=100$ to $D=1000 \mu \mathrm{m}^{2} / \mathrm{s}$. Notice that in water at $310 \mathrm{~K}, D \sim 10^{3} \mu \mathrm{m}^{2} / \mathrm{s}$ for small molecules and $D \sim 10 \mu \mathrm{m}^{2} / \mathrm{s}$ for large molecules [4]. The slot time $\tau$ and inter-symbol time $T$ are changed from $1 \times 10^{-4}$ to $2 \times 10^{-6} s$.

In Table I, the communication rates are presented according to changing values of diffusion coefficient $D$. Here, the communication rates are computed by setting $T, \tau$, and $d$ as $T=\tau=1 \times 10^{-4} s$ and $d=2 \mu \mathrm{m}$. As observed in the table, for this parameter setting, MARCO significantly outperforms the other communication models in terms of providing high molecular communication rate. Furthermore, MARCO does not require time synchronization.

In Table II, the comparisons are given with respect to changing slot time $\tau$ and inter-symbol time $T$. Here, $d$ and $D$ are set to $d=2$ and $D=1000$. The rate of Z-, binary, and 4-input 2 output channels decreases as the slot time decreases. This stems from the erroneous transmissions coming from the late molecules such that as the slot time decreases, the number of late molecules increases. On the other hand, the rate of MARCO can be improved by decreasing the intersymbol time and provide significantly higher communication rate than Z-, binary, and 4-input 2 output channel approaches. The comparisons according to changing values of inter-node distance, i.e., $d$, are presented in Table III. Here, $T, \tau$, and $D$ are set to $T=2 \times 10^{-5}, \tau=2 \times 10^{-3}$, and $D=1000$, 
TABLE III

Rate Comparison With Respect To VARying VAlues of Inter-Node Distance $(d, \mu m)$

\begin{tabular}{l|l|l|l|l|l|l|l|l|l|l}
\hline \hline Inter-Node Distance & $d=1$ & $d=2$ & $d=3$ & $d=4$ & $d=5$ & $d=6$ & $d=7$ & $d=8$ & $d=9$ & $d=10$ \\
\hline \hline Z-channel & 211.18 & 94.25 & 37.05 & 12.25 & 3.30 & 0.71 & 0.12 & 0.16 & 0.01 & 0.00 \\
\hline Binary Channel & 155.06 & 45.10 & 10.44 & 1.88 & 3.59 & 3.06 & 1.73 & 0.74 & 0.25 & 0.07 \\
\hline 4-input 2-output Channel & 90.08 & 42.54 & 23.48 & 13.70 & 7.60 & 3.75 & 1.60 & 0.59 & 0.19 & 0.05 \\
\hline MARCO Channel & 1913.80 & 1168.56 & 746.58 & 2783.86 & 2781.67 & 2780.48 & 2779.76 & 2779.30 & 2778.78 & 2778.78 \\
\hline
\end{tabular}

respectively. The rates of Z-, binary, and 4-input 2 output channel decrease as $d$ increases. The rate of MARCO also decreases as $d$ changes from $d=1$ to $d=3$. However, as $d$ is further increased, the rate of MARCO converges to nearly $2700(\mathrm{bps})$. This result shows that the rate of MARCO may become independent of the inter-node distance after some critical values of the inter-node distance. Furthermore, MARCO significantly outperforms Z-, binary, and 4-input 2 output channel approaches.

\section{CONCLUSION}

In this paper, we introduce the molecular array-based communication (MARCO) scheme that uses the transmission order of different molecules to encode and exchange information symbols between nanomachines in nanonetworks. In order to quantify the MARCO performance, we first extensively derive the inter-symbol interference (ISI) and error probabilities based on the principles of Brownian motion. Then, using these probabilities we give an achievable rate expression for the MARCO channel in which two distinct molecules are used to encode the information. Numerical results and performance comparisons clearly show that MARCO incomparably outperforms the previously developed molecular communication models. More specifically, it is revealed by the numerical results and performance comparisons that MARCO can provide significantly higher communication capacity, i.e., on the scale of $100 \mathrm{Kbps.}$, than the previously developed molecular communication models.

\section{APPENDIX}

In this section, we first introduce the Z-channel approach then, binary and 4-input 2-output channel approaches. As shown in Fig. 9(a), in the z-channel approach, a molecule is transmitted by TN at the beginning of the time slot to deliver logic 1 . If the molecule reaches $\mathrm{RN}$ within a slot duration, i.e., $\tau, \mathrm{RN}$ can correctly receive the logic bit 1 with the probability $\eta$. Otherwise, $\mathrm{RN}$ erroneously receives bit 0 with probability $(1-\eta)$. In order to deliver 0 , TN transmits no molecule within a slot duration and all 0 transmissions are assumed to be successful in the Z-channel [23]. Hence, the probability $\eta$ that TN successfully transmits bit 1 to $\mathrm{RN}$ can be obtained as

$$
\eta=\int_{0}^{\tau} f(t) d t=\int_{0}^{\tau} \frac{d}{\sqrt{4 \pi D t^{3}}} e^{-\frac{d^{2}}{4 D t}} d t
$$

Using $\eta$, the channel transition matrix of the Z-channel, i.e., $\mathbf{P}_{\mathbf{z}}(y \mid x)$, can be given as

$$
\mathbf{P}_{\mathbf{z}}(y \mid x)=\left[\begin{array}{cc}
1 & 0 \\
1-\eta & \eta
\end{array}\right]
$$

where $x$ and $y$ denote the channel input and output, respectively. Based on the transition matrix $\mathbf{P}_{\mathbf{z}}(y \mid x)$, the capacity of the $\mathrm{Z}$-channel, i.e., $C_{z}$, can be given as

$$
C_{z}=\max _{Q(x)} I(X ; Y)
$$

That is,

$$
C_{z}=\max _{Q(x)} \sum_{x \in \mathcal{X}} \sum_{y \in \mathcal{Y}} Q(x) p_{z}(y \mid x) \log _{2}\left(\frac{p_{z}(y \mid x)}{\sum_{x^{\prime} \in \mathcal{X}} p_{z}\left(y \mid x^{\prime}\right)}\right)
$$

where $I(X ; Y)$ is the mutual information of the Z-channel. $\mathcal{X}=\{0,1\}$ and $\mathcal{Y}=\{0,1\}$ are the input and output alphabets of the Z-channel, respectively. $Q(x)$ denotes the distribution of the input, i.e., $Q(0)=\operatorname{Pr}(x=0)$ and $Q(1)=\operatorname{Pr}(x=1)$. $C_{z}$ is in bits per transmission. Since a transmission lasts for a slot duration $\tau$, a communication rate for the Z-channel in bits per second (bps) can be expressed as $\frac{C_{z}}{\tau}$ (bps).

In the Z-channel approach, all transmissions of bit 0 via emitting no molecule are assumed to be successful. However, this assumption is not realistic since the molecules emitted in the previous intervals may reach $\mathrm{RN}$ later when 0 bit is transmitted, which can clearly result in erroneous transmission. Therefore, such erroneous transmissions of logic bit 0 must be taken into account by employing a binary channel in Fig. 9(b) as previously introduced in [15], [21], [22]. Assuming that current transmission of logic bit 0 may be only affected by the logic bit 1 transmitted in the previous interval, the probability $\gamma$, that a logic bit 0 is not successfully delivered to $\mathrm{RN}$ by $\mathrm{TN}$, can be derived as

$$
\gamma=Q(1) \int_{\tau}^{2 \tau} f(t) d t=Q(1) \int_{\tau}^{2 \tau} \frac{d}{\sqrt{4 \pi D t^{3}}} e^{-\frac{d^{2}}{4 D t}} d t
$$

By using $\gamma$ and $\eta$ derived in (43), the channel transition matrix of the binary channel in Fig. 9(b), i.e., $\mathbf{P}_{\mathbf{b}}(y \mid x)$, can be found as in (44). Based on $\mathbf{P}_{\mathbf{b}}(y \mid x)$, the capacity of the binary channel in bits per transmission, i.e., $C_{b}$, can be derived using the same steps followed in (45). Furthermore, a communication rate in bps for the binary channel can be also given as $\frac{C_{b}}{\tau}$. In order to mitigate the interference from the late molecules in the Z- and binary channel approaches, the 4-input 2-output discrete memoryless channel approach is introduced in [23] to comprehensively model the molecular communication channel as shown in Fig. 9(c). In this approach, the input and output alphabets are given as $\mathcal{X}=\{00,01,10,11\}$ and $\mathcal{Y}=\{0,1\}$, respectively. Furthermore, the channel transition probability $\alpha$ in Fig. 9(c) can be derived as follows:

$$
\alpha=\int_{\tau}^{2 \tau} f(t) d t=\int_{\tau}^{2 \tau} \frac{d}{\sqrt{4 \pi D t^{3}}} e^{-\frac{d^{2}}{4 D t}} d t
$$


Hence, using $\alpha$ and $\eta$ derived in (43), the channel transition matrix for the 4-input 2-output channel in Fig. 9(c), i.e., $\mathbf{P}_{\mathbf{f}}(y \mid x)$, can be computed as in (44). Based on $\mathbf{P}_{\mathbf{f}}(y \mid x)$, the capacity of 4-input 2-output channel in bits per transmission, i.e., $C_{f}$, can be derived using the same steps followed in (45). Note that since a transmission lasts for two slot duration, $2 \tau$, a communication rate in bps for 4-input 2-output channel can be given as $\frac{C_{f}}{2 \tau}$. Next, using the derived rate expression of Z-, binary, and 4-input 2-output channels, the performance comparisons of MARCO with these channel approaches are presented.

\section{REFERENCES}

[1] V. Balzani, A. Credi and M. Venturi, "Molecular devices and machines", Nanotoday, vol. 2, no. 2, pp. 18-25, April 2007.

[2] E. Drexler, "Engines of creation: The coming era of nanotechnology", Doubleday, New York, 1986.

[3] I. F. Akyildiz, F. Brunetti, C. Blazquez, "NanoNetworking: A New Communication Paradigm", Computer Networks Journal (Elsevier), June 2008.

[4] R. A. Freitas, "Nanomedicine, Volume I: Basic Capabilities", Landes Bioscience, Texas, USA, 1999.

[5] C. M.Waters, B. L. Bassler, "Quorum Sensing: Cell-to-Cell Communication in Bacteria", Annual Review of Cell and Developmental Biology, vol. 21, pp.319-346, Nov. 2005.

[6] T. A. Springer, "Adhesion receptors of the immune system", Nature, vol. 346, pp. 425-434, August 1990.

[7] M-T. Chen, R. Weiss, "Artificial cell-cell communication in yeast Saccharomyces cerevisiae using signaling elements from Arabidopsis thaliana”, Nature Biotechnology, vol. 23, pp. 1551-1555, 2005.

[8] S. Hiyama, Y. Moritani, T. Suda, R. Egashira, A. Enomoto, M. Moore and T. Nakano, "Molecular Communication", In Proc. of NSTI Nanotech 2005, Anaheim, California, USA, 2005.

[9] M. Pierobon, I. F. Akyildiz, "A Physical Channel Model for Molecular Communication in Nanonetworks", to appear in IEEE Journal of Selected Areas in Communications, 2010.

[10] K. V. Srinivas, R. S. Adve, A. W. Eckford, "Molecular communication in fluid media: The additive inverse Gaussian noise channel", submitted to IEEE Transactions on Information Theory, Dec. 2010.

[11] M. Pierobon, I. F. Akyildiz, "Diffusion-based Noise Analysis for Molecular Communication in Nanonetworks", IEEE Transactions on Signal Processing, vol. 59, no. 6, pp. 2532-2547, June 2011.

[12] M. Moore, T. Suda, K. Oiwa, "Molecular Communication: Modeling Noise Effects on Information Rate", IEEE Transactions on Nanobioscience, vol. 8, pp.169-180, June 2009.

[13] T. Nakano, L. Jian-Qin, "Design and Analysis of Molecular Relay Channels: An Information Theoretic Approach", IEEE Transactions on Nanobioscience, vol. 9, no. 3, pp. 213-221, Sept. 2010.

[14] T. Nakano, M. Moore, "In-sequence molecule delivery over an aqueous medium ", Nano Communication Networks (Elsevier),vol.1, no. 3, pp. 181-188, September 2010.

[15] B. Atakan, O. B. Akan, "On Channel Capacity and Error Compensation in Molecular Communication", Springer Trans. on Computational System Biology, vol. 10, pp. 59-80, February 2008.

[16] B. Atakan, O. B. Akan, "Deterministic capacity of information flow in molecular nanonetworks ", Nano Communication Networks (Elsevier),vol.1, no. 1, pp. 31-42, March 2010.

[17] B. Atakan, O. B. Akan, "On Molecular Multiple-Access, Broadcast and Relay Channels in Nanonetworks", in Proc. ACM BIONETICS 2008, Hyogo, Japan, December, 2008.

[18] B. Atakan, O. B. Akan, "Single and Multiple-Access Channel Capacity in Molecular Nanonetworks", in Proc. ICST/ACM Nano-Net 2009, Luzern, Switzerland, October 2009.

[19] V. Balzani, A. Credi, M. Venturi, "Molecular devices and machines: concepts and perspectives for the nanoworld", WILEY-VCH, 2008.

[20] I. Karatzas, S. E. Shreve, "Brownian Motion and Stochastic Calculus", Springer (New York), 1991.

[21] M. Ş. Kuran, H. B. Yılmaz, T. Tuğcu, B. Özerman, "Energy model for communication via diffusion in nanonetworks ", Nano Communication Networks (Elsevier),vol.1, no. 2, pp. 86-95, June 2010.
[22] M. U. Mahfuz, D. Makrakis, H. T. Mouftah, "On the characterization of binary concentration-encoded molecular communication in nanonetworks ", Nano Communication Networks (Elsevier),vol.1, no. 4, pp. 289300, Dec. 2010

[23] D. Arifler, "Capacity analysis of a diffusion-based short-range molecular nano-communication channel", Computer Networks (Elsevier),vol.55, no. 6, pp. 1426-1434, April 2011.

[24] A. W. Eckford, "Nanoscale Communication with Brownian Motion", in Proc. Information Sciences and Systems 2007, (CISS' 07), pp. 160-165, Baltimore, MD, September 2007.

Baris Atakan: Baris Atakan received the B.Sc. and M.Sc. degrees in electrical and electronics engineering from Ankara University and Middle East Technical University (METU), Ankara, Turkey, in 2000 and 2005, respectively. He received the Ph.D. degree in electrical and electronics engineering from the Next-generation and Wireless Communications Laboratory (NWCL), School of Sciences and Engineering, Koc University, Istanbul, in 2011. His current research interests include nanoscale and molecular communication, nanonetworks, and biologically-inspired communication protocols for wireless networks.

Sebastià Galmés: Sebastià Galmés received his degree of Electrical Engineer from the Polytechnic University of Catalonia (Barcelona, Spain) in 1989 and his $\mathrm{Ph}$. D. degree in Computer Science from the University of the Balearic Islands (Palma, Spain) in 1999, where he is currently Associate Professor in the Department of Mathematics and Computer Science. He was visiting professor at the North Carolina State University during academic year 1992-1993. His current research interests focus on wireless sensor networks, nanonetworks and cognitive radios. He is Area Editor of Simulation Modelling Practice and Theory and member of IFIP WG 6.3 and IEEE.

Ozgur B. Akan: OZGUR B. AKAN [M'00, SM'07] (akan@ku.edu.tr) received his Ph.D. degree in electrical and computer engineering from the Broadband and Wireless Networking Laboratory, School of Electrical and Computer Engineering, Georgia Institute of Technology in 2004. He is currently an associate professor with the Department of Electrical and Electronics Engineering, Koc University and the director of the Next-generation and Wireless Communications Laboratory. His current research interests are in wireless communications, nano-scale and molecular communications, and information theory. He is an Associate Editor of IEEE Transactions on Vehicular Technology, International Journal of Communication Systems (Wiley), and Nano Communication Networks Journal (Elsevier). He is currently General Co-Chair of ACM MOBICOM 2012, IEEE MoNaCom 2012, and TPC CoChair of IEEE ISCC 2012. 